\title{
Rhein induces liver cancer cells apoptosis via activating ROS-dependent JNK/Jun/caspase-3 signaling pathway
}

\author{
Aili Wang $1,2,3^{*}$, Huihong Jiang ${ }^{1,2,3^{*}}$, Yuanyuan Liu ${ }^{1,2,3}$, Jing Chen ${ }^{1,2,3}$, Xue Zhou $1,2,3$, Chenxi Zhao ${ }^{1,2,3}$, Xia \\ Chen ${ }^{1,2,3}$, Mobin Lin 1,2,3凶 \\ 1. Center for clinical research and translational medicine, Yangpu hospital, Tongji University School of Medicine, Shanghai 200090, China. \\ 2. Institute of Gastrointestinal Surgery and Translational Medicine, Tongii University School of Medicine, Shanghai 200090, China. \\ 3. Department of General Surgery, Yangpu Hospital, Tongji University School of Medicine, Shanghai 200090, China. \\ *These authors contributed equally to this work. \\ $\triangle$ Corresponding author: Department of General medicine, Yangpu Hospital of Tongji University, Department of General Practice of Tongji University, 450 \\ Tenyue Road, Shanghai 200090, China. Tel.: +86 21 65690520; fax: +86 21 65696249, E-mail address: 1500142@tongji.edu.cn. \\ (C) The author(s). This is an open access article distributed under the terms of the Creative Commons Attribution License (https://creativecommons.org/licenses/by/4.0/).
} See http://ivyspring.com/terms for full terms and conditions.

Received: 2018.10.03; Accepted: 2019.09.22; Published: 2020.01.01

\begin{abstract}
Background: Liver cancer is one of the leading cancers in China. Rhein induces apoptosis in various human cancer cells, but the underlying mechanism is still unknown.

Methods: In the present study, the MTT assay was used to detect the anti-cell growth ability of Rhein on liver cancer cells. Hoechst33342 staining and FACS assay were used to detect cell apoptosis. Finally, the effect of Rhein on JNK protein' phosphorylation level and the apoptosis-associated proteins were determined by western blot.

Results: Here, we found that Rhein significantly inhibited the cell viability in a dose-dependent and time-dependent manner both in HepG2 and Huh7 cells. Also, Rhein increased the apoptosis, mitochondrial membrane potential (MMP) and cell-cycle arrest. Furthermore, we observed that the ROS level and JNK/Jun/caspase-3 signaling pathway played a key role in Rhein induced apoptosis. Our study further demonstrated that Rhein increases apoptosis by inducing the generation of ROS and activating the JNK/Jun/caspase-3 signaling pathway.

Conclusions: The present study showed that Rhein promotes apoptosis via regulating ROS/JNK/Jun/caspase-3 signaling pathway both in HepG2 and Huh7 cells. Rhein may be a promising therapeutic candidate for the treatment of liver cancer.
\end{abstract}

Key words: Rhein; liver cancer; apoptosis; ROS; JNK/Jun/caspase-3 signaling pathway

\section{Introduction}

Liver cancer, the third cancer-related cause of death, is one of the leading cancers with a rapid upward trend all over the world. Epidemiology studies have shown that liver cancer is the second most common digestive cancer in Asia. In China, there is a large variation in the incidence, with high incidence rates in men of more than 30 per 100,000 [1]. However, no chemotherapeutics have been evidenced better efficacious therapies and prevention strategies for liver cancer [1]. Therefore, the identification of novel bioactive compounds with promising anticancer activity and investigation of their cellular targets remains needed.
Rhein (4,5-dihydroxyanthraquinone-2-carboxylic acid, Figure 1A), a main constituent of rhubarb, is found in medicinal herbs including rheum palmatum 1 , cassia tora 1 , polygonum multiflorum thunb, aloe barbadensis miller, and so on. Rhein has been used medicinally for more than 1,000 years in China for the treatment of inflammatory diseases including osteoarthritis, diabetic nephropathy. The recent studies have demonstrated that Rhein induces significant apoptosis in human breast, colon, lung, and glioma cancer cell lines in vitro [2]. Apoptosis, a physiological process for eliminating malignant cells including cancer cells, plays a key role in anticancer 
without damaging normal cells and tissues [3, 4]. Previously, the substantial evidence showed that Rhein induces the cell cycle S-phase arrest and results in DNA fragmentation via complex mechanisms including decreasing $\mathrm{Bcl}-2$ and cleaved caspase-3 level and increasing ROS and phosphorylated c-JNK level $[2,5,6]$. These results suggest that Rhein induces apoptosis in various human cancer cells. However, whether Rhein is effective in killing liver cancer cells is still unknown.

Based on the above considerations, the aim of our present study is to investigate the potential anticancer effects of Rhein on hepatoma cells including HepG2 and Huh7 cells, and to further explore the underlying molecular mechanism of Rhein in the treatment of liver cancer. In this paper, we have provided the first evidence that Rhein promotes apoptosis through regulating ROS/JNK/ Jun/caspase-3 signaling pathway.

\section{Materials and methods}

\section{Drugs}

Rhein was purchased from the Chinese National Institute. It was dissolved in DMSO, and was added into the culture medium at the indicated concentrations (with a final DMSO concentration less than $0.1 \%)$.

\section{Cell culture}

HepG2 and Huh7 cells were obtained from the Cell Bank of Chinese Academy of Sciences (Shanghai, China). Cells were cultured with DMEM medium containing 10\% FBS and antibiotics (100 U/mL penicillin and $100 \mathrm{mg} / \mathrm{mL}$ streptomycin) in $\mathrm{CO}_{2}$ incubator (at $\left.37^{\circ} \mathrm{C}\right)$.

\section{MTT analysis}

Cells were treated with Rhein $(0,50,100,150$, and $200 \mu \mathrm{mol} / \mathrm{L}$ ) and cultured for $24 \mathrm{~h}, 48 \mathrm{~h}$, and $72 \mathrm{~h}$, respectively. After exposure to different concentrations of Rhein, the cell viability was detected with MTT analysis. Details of MTT analysis were in compliance with the previously described [7].

\section{Hoechst staining analysis}

Cells were treated with Rhein $(0,100,150$, and $200 \mu \mathrm{mol} / \mathrm{L})$ for $24 \mathrm{~h}$ in 96-well culture plates. Hoechst staining analysis was performed as described previously [8].The stained cells were observed with fluorescence-inverted microscopy (IX73; Olympus, Tokyo, Japan).

\section{TUNEL staining}

Cells were treated with Rhein $(0,100,150$, and $200 \mu \mathrm{mol} / \mathrm{L})$ for $24 \mathrm{~h}$. For in situ apoptosis detection, the cells were stained using TUNEL reagent according to the manufacturer's instructions. TUNEL-positive cells were analyzed under a fluorescence microscope. The data analysis of TUNEL staining was conducted as described previously [9].

\section{ROS level analysis}

ROS level was evaluated using ROS assay kit based on 2',7'-Dichlorodihydrofluorescin diacetate (DCFH-DA). Cells were treated with Rhein $(0,100$, 150, and $200 \mu \mathrm{mol} / \mathrm{L}$ ) for $24 \mathrm{~h}$, and then incubated with DCFH-DA $(50 \mu \mathrm{mol} / \mathrm{L})$ for $30 \mathrm{~min}$ in the dark. ROS level analysis was performed as described previously [8].

\section{MMP level analysis}

MMP level was measured with JC-1 staining. Cells were treated with Rhein $(0,100,150$, and 200 $\mu \mathrm{mol} / \mathrm{L})$ and CCCP $(10 \mu \mathrm{mol} / \mathrm{L}$, as the positive control) for $24 \mathrm{~h}$, respectively. Then, the cells were stained with JC-1 reagent $(10 \mu \mathrm{g} / \mathrm{mL})$ at $37^{\circ} \mathrm{C}$ for 20 min. The result was analyzed by a flow cytometer (Becton Dickinson, USA). MMP level analysis was performed as described previously [10].

\section{Apoptosis and cell-cycle arrest analysis}

The apoptosis and cell-cycle arrest analysis were performed by FACS. Cells were treated with Rhein $(150 \mu \mathrm{mol} / \mathrm{L})$ or NAC $(1 \mathrm{mmol} / \mathrm{L})$ for $24 \mathrm{~h}$, and then were stained by annexin V-APC in conjunction with propidium iodide (PI). The detail of apoptosis and cell-cycle arrest analysis was conducted as described previously [11].

\section{Western blot analysis}

Western blot analysis was conducted as described previously [12]. Briefly, the total proteins were extracted with RIPA buffer (Beyotime, China). Protein concentrations were measured using enhanced BCA protein Assay kit (Beyotime, China) by spectrophotometer. Equal amounts of protein $(50 \mu \mathrm{g})$ were separated using $10 \%$ sodium dodecyl sulfate polyacrylamide gel electrophoresis (SDS-PAGE), were transferred onto PVDF membrane, and then were blocked with 5\% fat-free dry milk at room temperature for $1 \mathrm{~h}$. The membranes were incubated with primary antibodies including p-JNK(1:1000), JNK(1:1000), p-c-Jun(1:1000), c-Jun(1:1000), cleaved caspase-3(1:1000), caspase-3(1:1000) and $\beta$-actin (1:2000) at $4^{\circ} \mathrm{C}$ overnight, respectively. The next day, the membranes were washed using TBST washing buffer, and then incubated with the peroxidaseconjugated secondary antibody (1:5000) for $1 \mathrm{~h}$ at room temperature. After washed with TBST, the membranes were developed using ECL plus chemiluminescence kit on a DNR bio-imaging system 
MicroChemi. $\beta$-actin was used as an internal control to normalize results. The images were quantified using Image J software.

\section{Statistical analysis}

All data were presented as mean \pm SD. The GraphPad prism5.0 Software was used for statistical analysis. Student's $t$-test was used for the comparison between two samples, and two-way ANOVA analysis for two groups. A $P<0.05$ was considered as statistical significance.

\section{Results}

\section{Rhein increased the apoptosis of HepG2 and Huh7 cells}

To investigate the effects of Rhein on the viability of HepG2 and Huh7 cells, we first examined the cell viability using MTT assay. The cells were treated with Rhein in a concentration gradient $(0,50$, 100, 150 and $200 \mu \mathrm{mol} / \mathrm{L})$ for 24,48 and $72 \mathrm{~h}$, respectively. As shown in Figure 1B, Rhein significantly increased cell death in a dose-dependent and time-dependent manner. The higher concentration of Rhein for $72 \mathrm{~h}$ has a stronger pro-apoptotic effect. These results suggest that Rhein induces cell apoptosis both in HepG2 and Huh7 cells.

To further investigate the effect of Rhein on

A

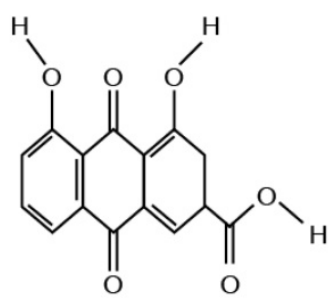

HepG2 and Huh7 cells, we tested the role of Rhein (0, 100, 150 and $200 \mu \mathrm{mol} / \mathrm{L}$ for $24 \mathrm{~h}$ ) on apoptotic morphological characteristics with Hoechst 33342 staining. Our data showed that Rhein increased the condensation and fragmentation of nuclei, indicating that the drug enhances cell apoptosis (Figure 1C and D). Altogether, these results suggest that Rhein promotes cell apoptosis of liver cancer cells in vitro.

\section{The effects of Rhein on the MMP}

Previously, it has been demonstrated that MMP is one of the main characteristics of early apoptosis of cells [13]. To investigate whether mitochondria are involved in Rhein-induced apoptosis, we measured MMP of cells using JC-1 staining. As shown in Figure 2A, Rhein exhibited a significant loss of MMP in a dose-dependent manner compared with the control group, close to the effects of CCCP (a widely used reagent to induce cell apoptosis) treatment. In addition, we also analyzed DNA fragmentation using the TUNEL staining, a widely used method to determine cell apoptosis. Our results showed that the cells of Rhein group exhibited elevated doublestranded DNA breaks compared with the control group both in HepG2 and Huh7 cells (Figure 2B). Altogether, these results suggest that Rhein increases the apoptosis of HepG2 and Huh7 cells.

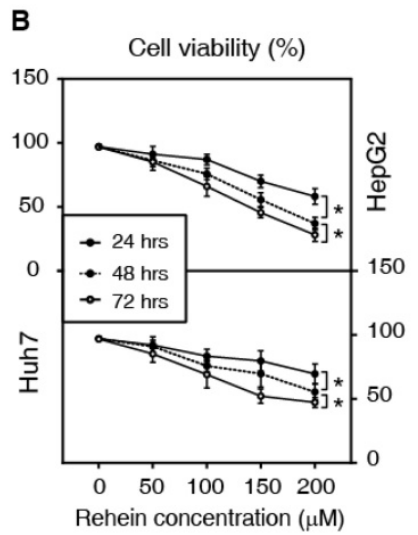

C

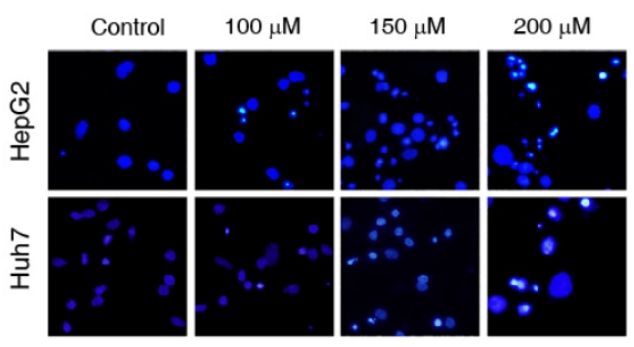

D

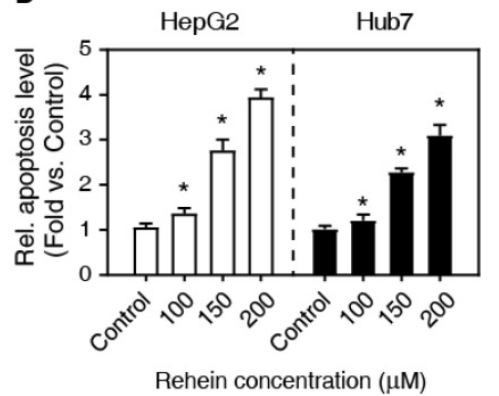

Figure 1. Rhein increased the apoptosis of HepG2 and Huh7 cells. (A) The chemical structure of Rhein. (B) HepG2 and Huh7 cells were treated with Rhein (0, 50 , $100,150$ and $200 \mu \mathrm{mol} / \mathrm{L})$ for 24,48 and $72 \mathrm{~h}$, respectively. Cell viability was measured by MTT assay. *P < 0.05 . (C, D) HepG2 and Huh7 cells were treated with Rhein $(0,100,150$ and $200 \mu \mathrm{mol} / \mathrm{L})$ for $24 \mathrm{~h}$, and then the apoptotic morphological characteristics were stained with Hoechst 33342 staining. $* P<0.05$ compared with the control group. 
A

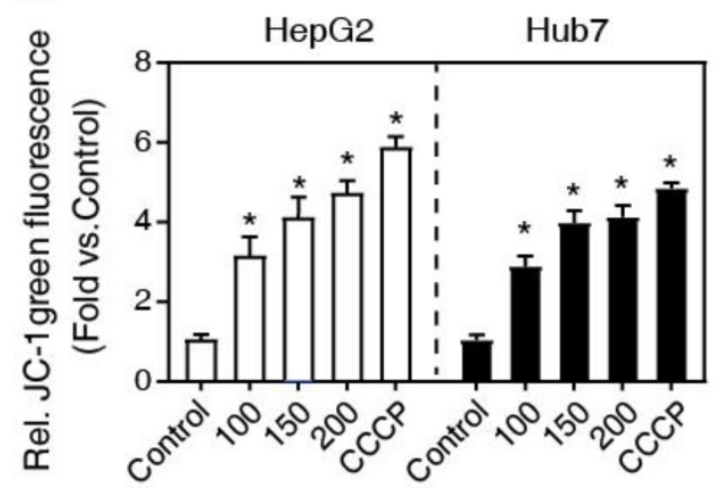

Rehein concentration $(\mu \mathrm{M})$
B
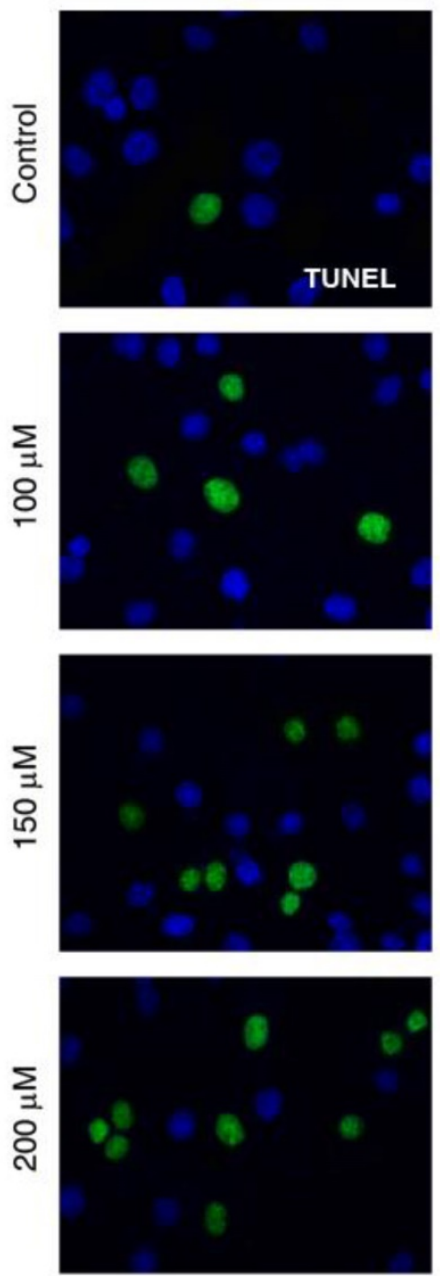

Huh7
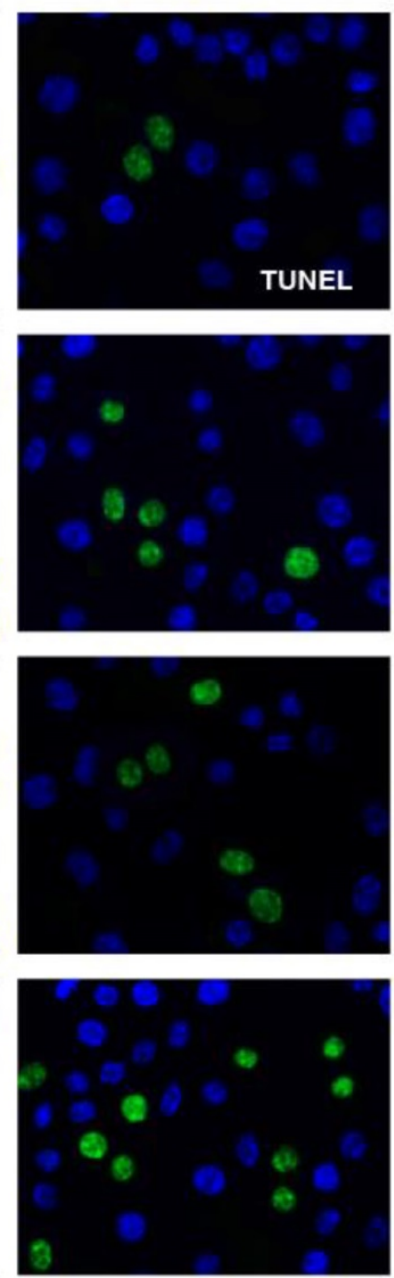

Rehein concentration $(\mu \mathrm{M})$

Figure 2. The effects of Rhein on the MMP. (A) HepG 2 and Huh7 cells were treated with Rhein (0, 100, 150 and $200 \mu \mathrm{mol} / \mathrm{L})$ or CCCP (10 $\mu \mathrm{mol} / \mathrm{L})$. The MMP was measured with flow cytometer using JC-1 staining. (B) HepG2 and Huh7 cells were treated with Rhein (0,100, 150 and $200 \mu \mathrm{mol} / \mathrm{L})$ for $24 \mathrm{~h}$, and then the DNA fragmentation was detected using a TUNEL kit. (C) HepG2 and Huh7 cells were treated with Rhein $(0,100,150$ and $200 \mu \mathrm{mol} / \mathrm{L})$ for $24 \mathrm{~h}$. The ROS level was measured with DCFH-DA. $* P<0.05$ compared with the control group.

\section{Rhein increases the level of ROS.}

It is well known that ROS is a pro-apoptosis factor $[13,14]$. To determine whether ROS is associated with the role of Rhein on apoptosis in HepG2 and Huh7 cells, we examined the regulatory role of Rhein $(0,100,150$ and $200 \mu \mathrm{mol} / \mathrm{L}$ for $24 \mathrm{~h})$ on ROS level using DCFH-DA-based assay. As shown in Figure 2C, Rhein significantly increased the ROS level in a dose-dependent manner, suggesting that Rhein induces the generation of ROS. To confirm that Rhein increases apoptosis through regulating the ROS level, we pre-treated cells with NAC, a widely used ROS scavenger. Interestingly, NAC obviously abolished the cell apoptosis induced by Rhein (Figure 3A-C). Conclusively, these data suggest that Rhein induces apoptosis via regulating the level of ROS in HepG2 and Huh7 cells. Next, we analyzed the effect of Rhein on the cell-cycle progression by FACS. As shown in Figure 3D, Rhein induced a marked G0/G1 arrest both in HepG2 and Huh7 cells, and these effects were abolished by NAC pre-treatment. These results collectively indicate that Rhein induces cell apoptosis and cell-cycle arrest via regulating the ROS level in vitro.

\section{Rhein induced apoptosis via regulating the JNK/Jun/caspase-3 signaling pathway}

Previous studies have shown that ROS activates the JNK kinase, and the activated JNK subsequently phosphorylates its substrate, c-Jun, and the phosphorylated c-Jun (p-c-Jun) further induces the activation of caspase-3 protein [15] (Figure 5C). Therefore, we speculated that Rhein induces cell apoptosis by regulating the JNK/Jun/caspase-3 signaling pathway. Then, we firstly detected the 
expression of phosphorylated JNK (p-JNK) using western blotting. As shown in Figure 4A and B, Rhein increased p-JNK level both in HepG2 and Huh7 cells. Meanwhile, NAC pretreatment significantly blocked the elevated p-JNK level induced by Rhein. Moreover, our findings further showed that Rhein also significantly increased the phosphorylation of c-Jun (p-c-Jun) (Figure 4C and D) and the cleaved caspase-3 protein (c-caspase-3) level (Figure 5A and B), and these effects of Rhein were obviously reversed by NAC pre-treatment. Taken together, our observations suggest that Rhein-induced ROS activates the JNK/Jun/caspase-3 signaling pathway, and then induces cell apoptosis in vitro.

\section{Discussion}

Liver cancer is an untreatable solid tumor. After long-term therapy, many patients of advanced liver cancer eventually become treatment-resistant. It was previously reported that the inhibition of cell apoptosis plays a vital role in the pathogenesis of cancers [16]. Therefore, promoting cell apoptosis has been a critical strategy for antitumor therapy during the past several decades [17, 18]. Emerging evidence indicates that the up-regulation of ROS level is required for the initiation of apoptotic responses induced by several anticancer agents. ROS mediates many physiological and pathological progress in cells via JNK signaling pathway. The activated JNK kinase phosphorylates the c-Jun protein, by which c-Jun initiates the downstream apoptotic events including the cleavage of caspase-3 [19-21]. Thus, ROS/JNK/ c-Jun/caspase-3 signaling has been recognized as an important pathway for developing anticancer drugs.
A
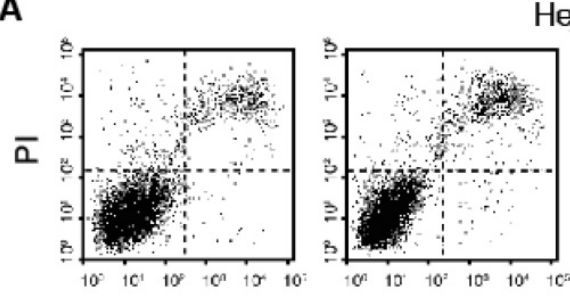

HepG2

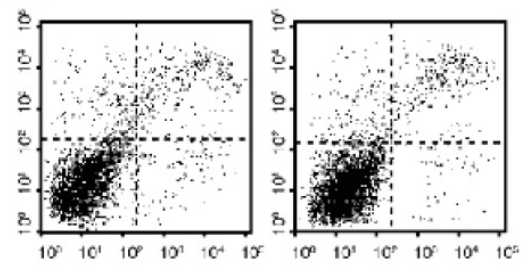

B

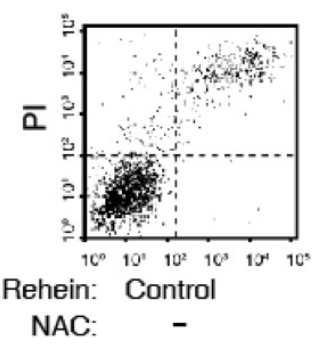

Huh7
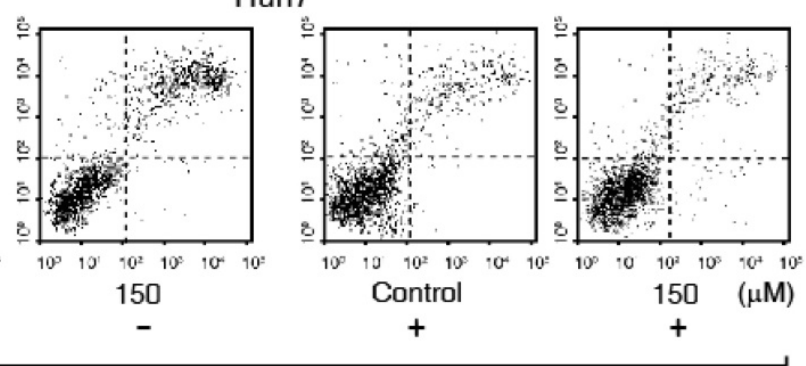

Annexin-V

C
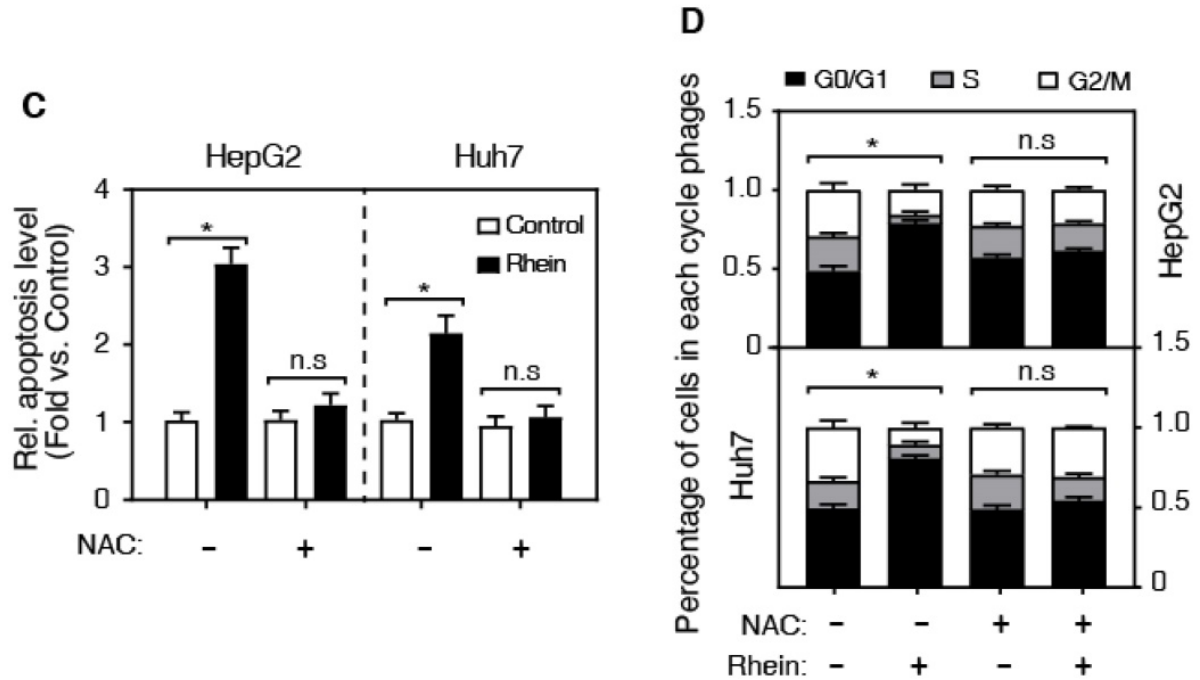

Figure 3. Rhein increases cell apoptosis and cell-cycle arrest. (A-C) The effect of NAC on Rhein-induced cell apoptosis. HepG2 and Huh7 cells were treated with control or Rhein, and then incubated with NAC. (D) The effect of NAC on Rhein-induced the cell-cycle arrest. $* P<0.05$; n.s, not statistically significant. 

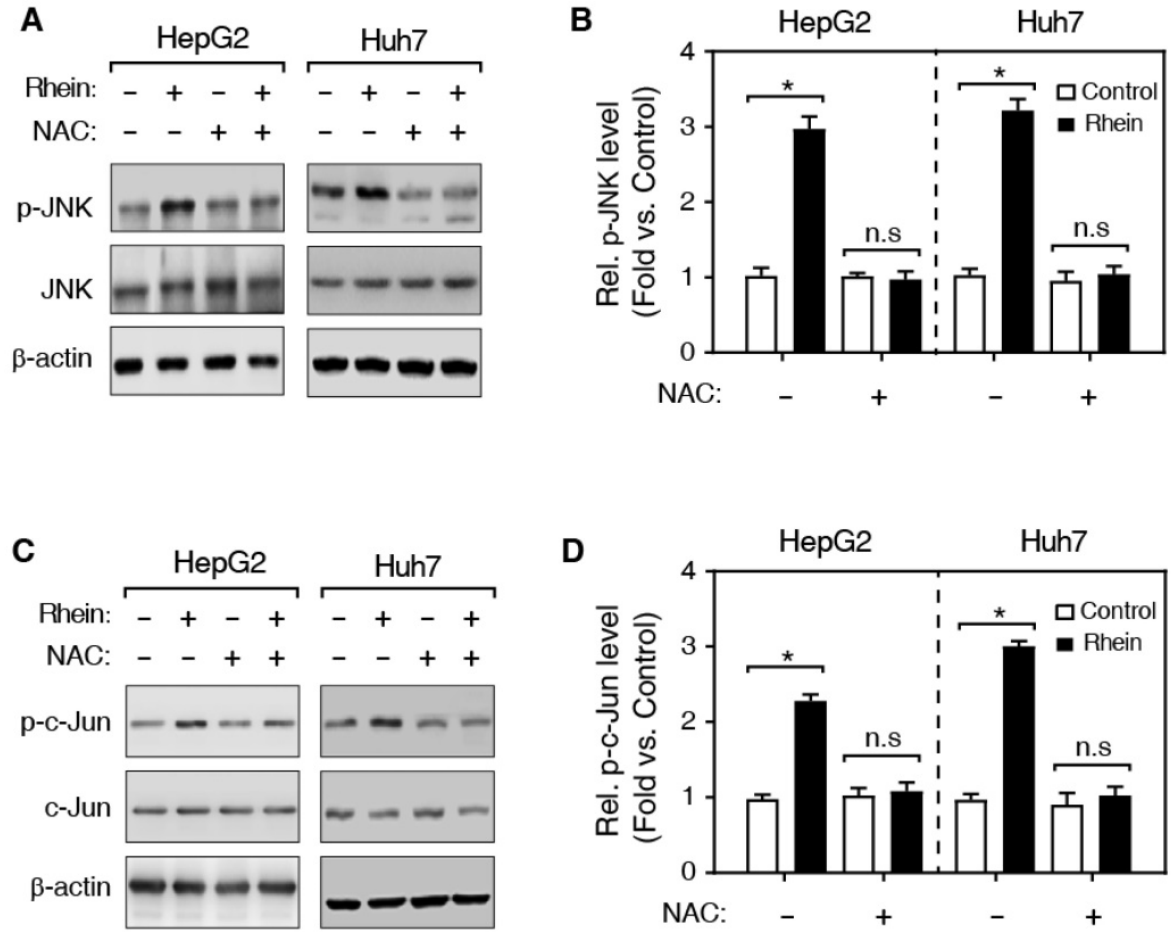

Figure 4. Effects of Rhein on the phosphorylation of JNK and c-Jun. (A, C) Western blot determined expression levels of JNK, p-JNK (A) and c-Jun, p-c-Jun (C). HepG2 and Huh7 cells were treated with/without Rhein, and then incubated with/without NAC. $\beta$-actin served as the loading control. (B, D) The quantification of protein levels in $A$ and $C$. *P $<0.05 ;$ n.s, not statistically significant.

A
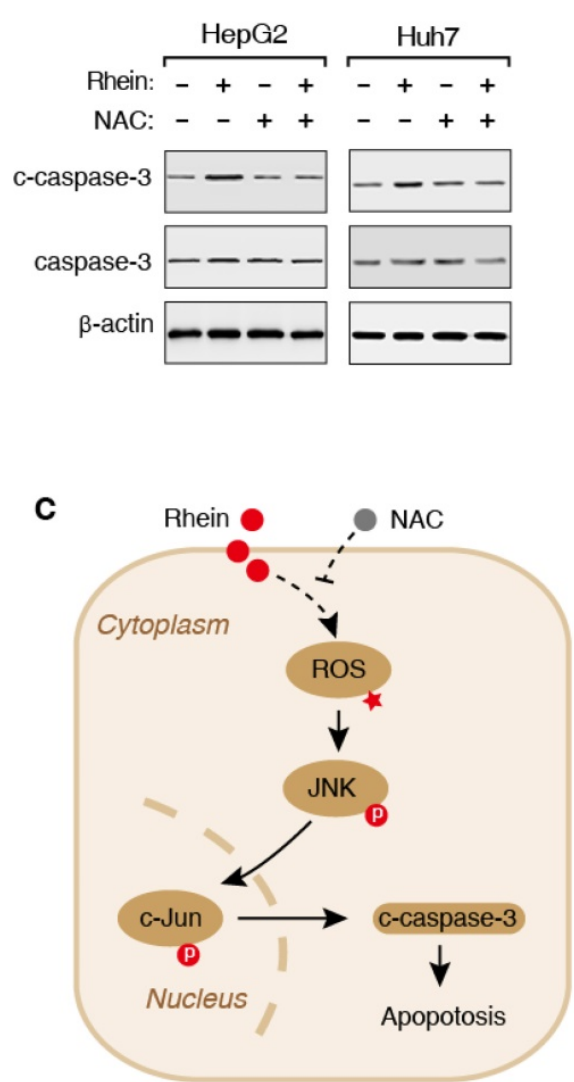

\section{B}

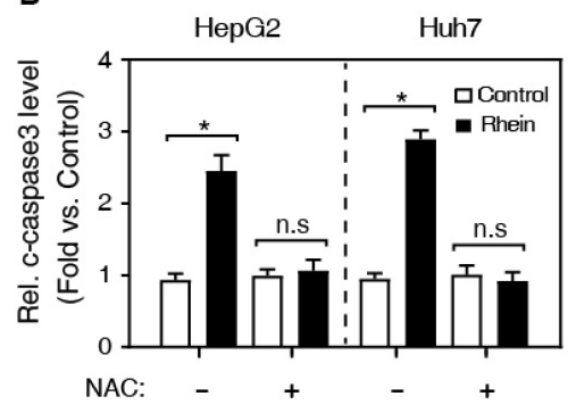

Figure 5. Effects of Rhein on the expression of cleaved caspase-3. (A) Western blot determined the expression of caspase-3 and c-caspase-3. HepG2 and Huh7 cells were treated with/without Rhein, and then incubated with/without NAC. $\beta$-actin served as the loading control. (B) The quantification of the c-caspase-3 protein level in A. ${ }^{* P}<0.05$; n.s, no significance in statistic. (C) The schematic representative of work model. Rhein induces the generation of ROS, and then activates the JNK/Jun/caspase-3 signaling pathway to promotes cell apoptosis. NAC, N-acetylcysteine; ROS, reactive oxygen species; JNK, c-Jun N-terminal kinase; $\mathrm{p}$, phosphorylation modification. 
In the present study, we found that Rhein induced an obvious nuclei condensation and fragmentation (a typical characteristic of apoptotic cells) in HepG2 and Huh7 cells in a dose- and time-dependent manner, indicating Rhein possesses a significant pro-apoptotic effect. Furthermore, our data showed that Rhein induced double-stranded DNA breaks in HepG2 and Huh7 cells, determined by the TUNEL staining. We also observed that Rhein induced early cell apoptosis, indicated by a significant loss of MMP in vitro. These findings suggest that Rhein increases cell apoptosis both in HepG2 and Huh7 cells. Meanwhile, we determined the ROS level in cells, and found that Rhein significantly increased ROS level in HepG2 and Huh7 cells. More importantly, scavenging ROS by NAC pre-treatment obviously alleviated the cell apoptosis induced by Rhein. Besides, Rhein induced a marked G0/G1 arrest in HepG2 and Huh7 cells, which also was reversed by NAC pre-treatment. In brief, these results demonstrate that Rhein induces cell apoptosis and cell-cycle arrest by regulating ROS level. In our knowledge, we demonstrated, for the first time, that Rhein induces cell apoptosis in the context of liver cancer cells.

It was previously reported that ROS level in cells affects the activity of many signaling pathways, including JNK signaling pathway [22]. The activated JNK kinase phosphorylates c-Jun protein, and finally leads to the cell apoptosis in a caspase- 3 dependent manner [23]. In this study, we firstly found that Rhein increased the phosphorylation of JNK both in HepG2 and Huh7 cells. At the same time, NAC significantly inhibited the over-expression of p-JNK induced by Rhein. Also, our result showed that Rhein significantly increased the p-c-Jun and the c-caspase-3 protein, and the effects of Rhein were obviously reversed by NAC pre-treatment both in HepG2 and Huh7 cells. These results suggest that Rhein-induced ROS activates the JNK/Jun/caspase-3 signaling pathway, and then induces apoptosis in vitro.

In fact, some previous studies reported that Rhein increases the expression of p53, p21/WAF1, CD95, and its two forms of ligands in HepG2 cells. Besides, Rhein also reduces the expression of Bcl-2 and $\mathrm{Bcl}-\mathrm{XL}$, and increases the expression of Bax and Bak. Many independent groups have also reported that Rhein activates caspase-1, -3, -8, -9, and -12 . Altogether, these results suggest that the roles of Rhein in antitumor involve multiple pathways, and the pharmacological mechanisms of Rhein in liver cancer need further studies.

Taken together, our observations demonstrated that Rhein increases apoptosis through inducing the generation of ROS, and then activates
JNK/Jun/caspase-3 signaling (Figure 5C). Overall, the present study has provided the first evidence that Rhein promotes apoptosis via regulating ROS/JNK/Jun/caspase-3 signaling pathway both in HepG2 and Huh7 cells. These results may contribute to understanding the roles of Rhein in apoptosis, and developing new therapeutic approaches for liver cancer treatment.

\section{Abbreviations}

ROS: reactive oxygen species; NAC: $\mathrm{N}$-acetylcysteine; MMP: mitochondrial membrane potential; JNK: c-Jun N-terminal kinase.

\section{Acknowledgement}

This work was sponsored by the National Natural Science Foundation of China (81874201).

\section{Competing Interests}

The authors have declared that no competing interest exists.

\section{References}

1. Ko KP, Shin A, Cho S, Park SK, Yoo KY. Environmental contributions to gastrointestinal and liver cancer in the Asia-Pacific region. J Gastroenterol Hepatol. 2018; 33: 111-20.

2. Sun H, Luo G, Chen D, Xiang Z. A Comprehensive and System Review for the Pharmacological Mechanism of Action of Rhein, an Active Anthraquinone Ingredient. Front Pharmacol. 2016; 7: 247.

3. Wu C, Cao H, Zhou H, Sun L, Xue J, Li J, et al. Research Progress on the Antitumor Effects of Rhein: Literature Review. Anticancer Agents Med Chem. 2017; 17: 1624-32.

4. Li-Weber M. Targeting apoptosis pathways in cancer by Chinese medicine. Cancer Lett. 2013; 332: 304-12.

5. Zhou YX, Xia W, Yue W, Peng C, Rahman K, Zhang H. Rhein: A Review of Pharmacological Activities. Evid Based Complement Alternat Med. 2015; 2015: 578107.

6. Ye MY, Yao GY, Wei JC, Pan YM, Liao ZX, Wang HS. Synthesis, cytotoxicity, DNA binding and apoptosis of rhein-phosphonate derivatives as antitumor agents. Int J Mol Sci. 2013; 14: 9424-39.

7. Shi P, Huang Z, Chen G. Rhein induces apoptosis and cell cycle arrest in human hepatocellular carcinoma BEL-7402 cells. Am J Chin Med. 2008; 36: $805-13$.

8. Mehmood T, Maryam A, Tian X, Khan M, Ma T. Santamarine Inhibits NF-small ka, CyrillicB and STAT3 Activation and Induces Apoptosis in HepG2 Liver Cancer Cells via Oxidative Stress. J Cancer. 2017; 8: 3707-17.

9. Stubenhaus B, Pellettieri J. Detection of Apoptotic Cells in Planarians by Whole-Mount TUNEL. Methods Mol Biol. 2018; 1774: 435-44.

10. Song L, Wang Z, Wang Y, Guo D, Yang J, Chen L, et al. Natural Cyclopeptide RA-XII, a New Autophagy Inhibitor, Suppresses Protective Autophagy for Enhancing Apoptosis through AMPK/mTOR/P70S6K Pathways in HepG2 Cells. Molecules. 2017; 22: 1934.

11. Zhang T, Ikejima T, Li L, Wu R, Yuan X, Zhao J, et al. Impairment of Mitochondrial Biogenesis and Dynamics Involved in Isoniazid-Induced Apoptosis of HepG2 Cells Was Alleviated by p38 MAPK Pathway. Front Pharmacol. 2017: 8: 753.

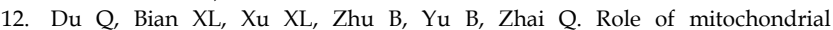
permeability transition in human hepatocellular carcinoma Hep-G2 cell death induced by rhein. Fitoterapia. 2013; 91: 68-73.

13. Li HY, Zhang J, Sun LL, Li BH, Gao HL, Xie T, et al. Celastrol induces apoptosis and autophagy via the ROS/JNK signaling pathway in human osteosarcoma cells: an in vitro and in vivo study. Cell Death Dis. 2015; 6: e1604.

14. Thongsom S, Suginta W, Lee KJ, Choe H, Talabnin C. Piperlongumine induces G2/M phase arrest and apoptosis in cholangiocarcinoma cells through the ROS-JNK-ERK signaling pathway. Apoptosis. 2017; 22: 1473-84.

15. Wang Y, Guo SH, Shang XJ, Yu LS, Zhu JW, Zhao A, et al. Triptolide induces Sertoli cell apoptosis in mice via ROS/JNK-dependent activation of the mitochondrial pathway and inhibition of Nrf2-mediated antioxidant response. Acta Pharmacol Sin. 2018; 39: 311-27. 
16. Sia D, Villanueva A, Friedman SL, Llovet JM. Liver Cancer Cell of Origin, Molecular Class, and Effects on Patient Prognosis. Gastroenterology. 2017; 152: 745-61.

17. Lin S, Zhang YJ. Interference of Apoptosis by Hepatitis B Virus. Viruses. 2017; 9: e230.

18. Mohamed MS, Bishr MK, Almutairi FM, Ali AG. Inhibitors of apoptosis: clinical implications in cancer. Apoptosis. 2017; 22: 1487-509.

19. Liu W, Deng J, Dong F, Huo T, Wang Y, Wang L. Apoptosis induced by chrysotile from Sichuan Xinkang via ROS/JNK pathway in A549 cells. Xi Bao Yu Fen Zi Mian Yi Xue Za Zhi. 2017; 33: 1217-22.

20. Feng J, Yan PF, Zhao HY, Zhang FC, Zhao WH, Feng M. Inhibitor of Nicotinamide Phosphoribosyltransferase Sensitizes Glioblastoma Cells to Temozolomide via Activating ROS/JNK Signaling Pathway. Biomed Res Int. 2016; 2016: 1450843

21. Park MH, Kim JH, Chung YH, Lee SH. Bakuchiol sensitizes cancer cells to TRAIL through ROS- and JNK-mediated upregulation of death receptors and downregulation of survival proteins. Biochem Biophys Res Commun. 2016; 473: 586-92.

22. Zhou G, Yang Z, Wang X, Tao R, Zhou Y. TRAIL Enhances Shikonin Induced Apoptosis through ROS/JNK Signaling in Cholangiocarcinoma Cells. Cell Physiol Biochem. 2017; 42: 1073-86.

23. Sun ZL, Dong JL, Wu J. Juglanin induces apoptosis and autophagy in human breast cancer progression via ROS/JNK promotion. Biomed Pharmacother. 2017; 85: 303-12. 